Supporting Information

\title{
Preparation of anisotropic and oriented particles on a flexible
}

\section{substrate}

Mahshid Chekini, ${ }^{a}$ Ugo Cataldi, ${ }^{a}$ Plinio Maroni, ${ }^{b}$ Laure Guénée, ${ }^{c}$ Radovan Černý ${ }^{c}$ and Thomas Bürgi ${ }^{\text {a } *}$

Corresponding author email: Thomas.Buergi@unige.ch 

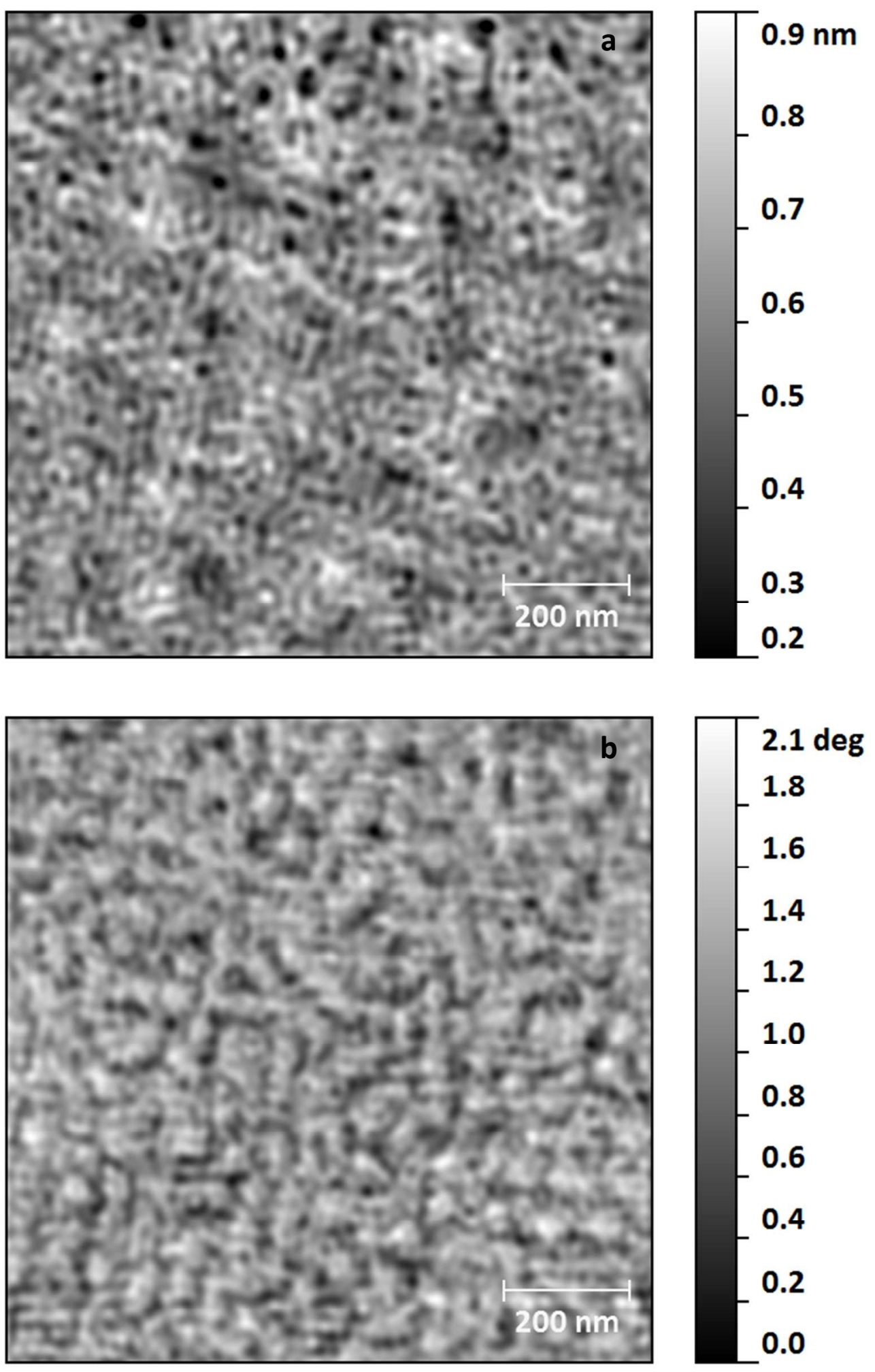


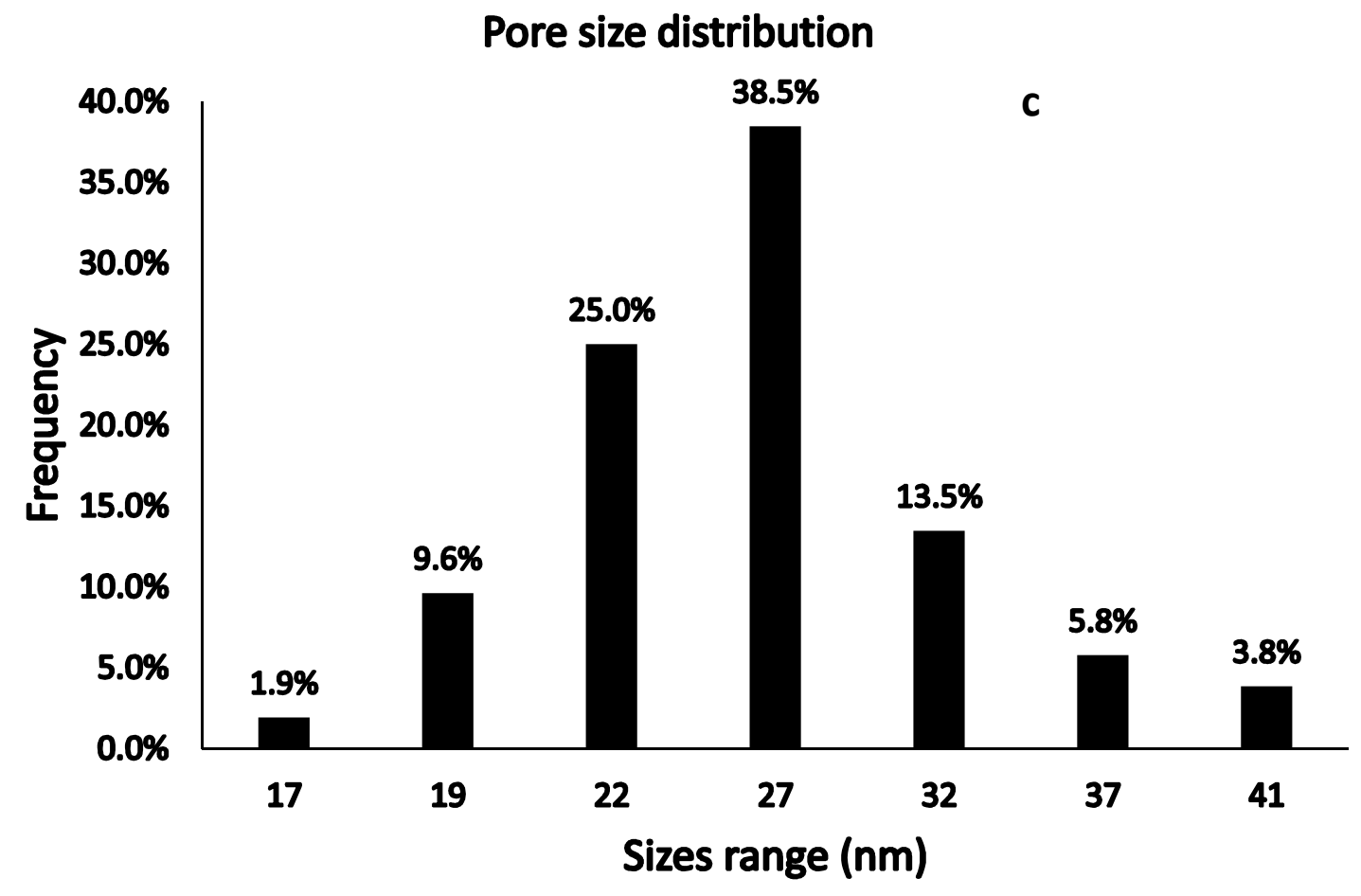

S 1 The AFM image of oxidized PDMS surface (after plasma treatment) analyzed by larger tip cantilever (Olympus OMCLAC240TS cantilever with a $9 \pm 2 \mathrm{~nm}$ tip) at a and its phase image at b Pore size distribution histogram calculated from image a at c. 

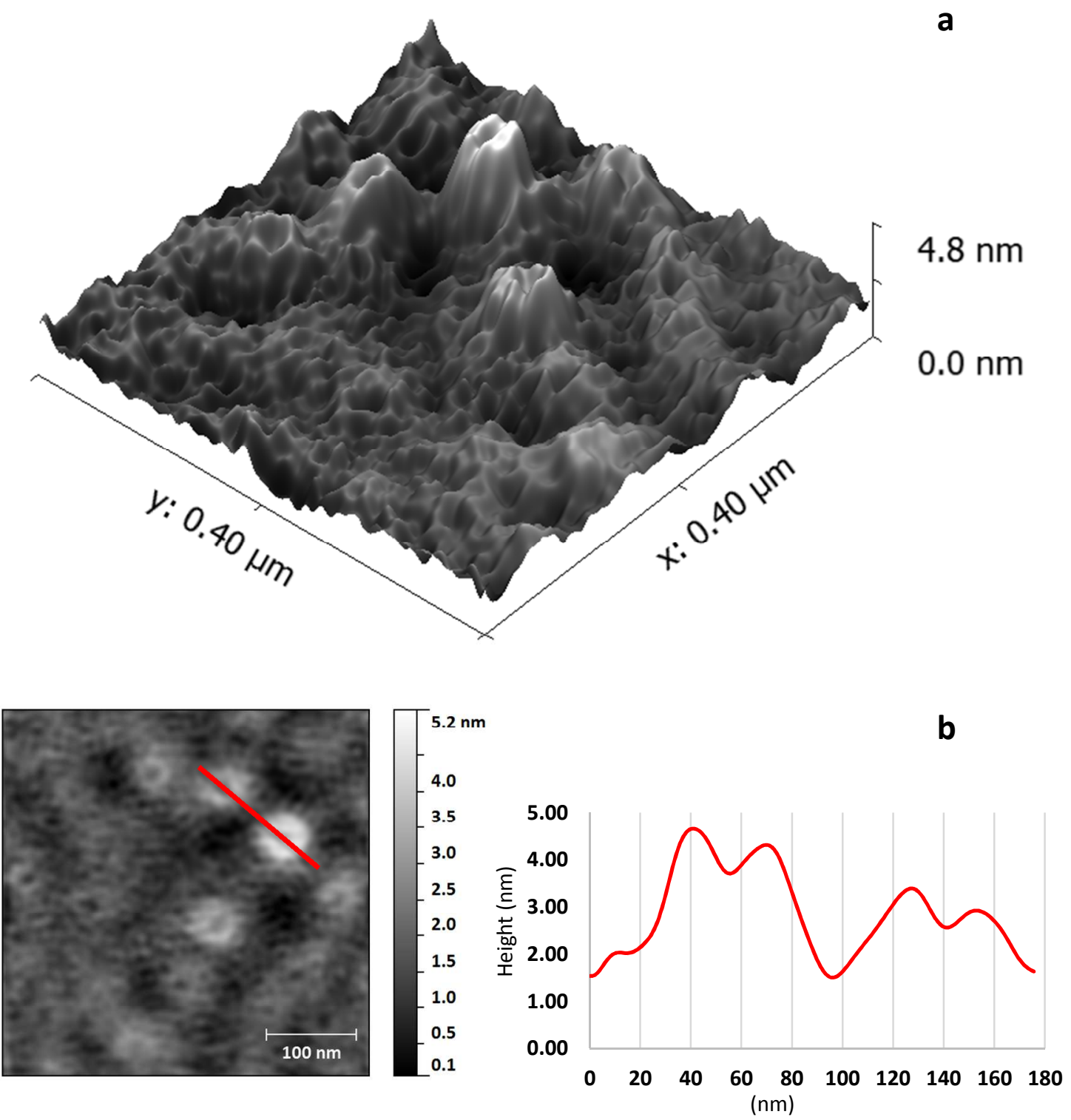

S2 3D-representation of Figure $4 \mathrm{e}$ at $\mathrm{a}$ and the plotted height profile along the red line presented at $\mathrm{b}$. 

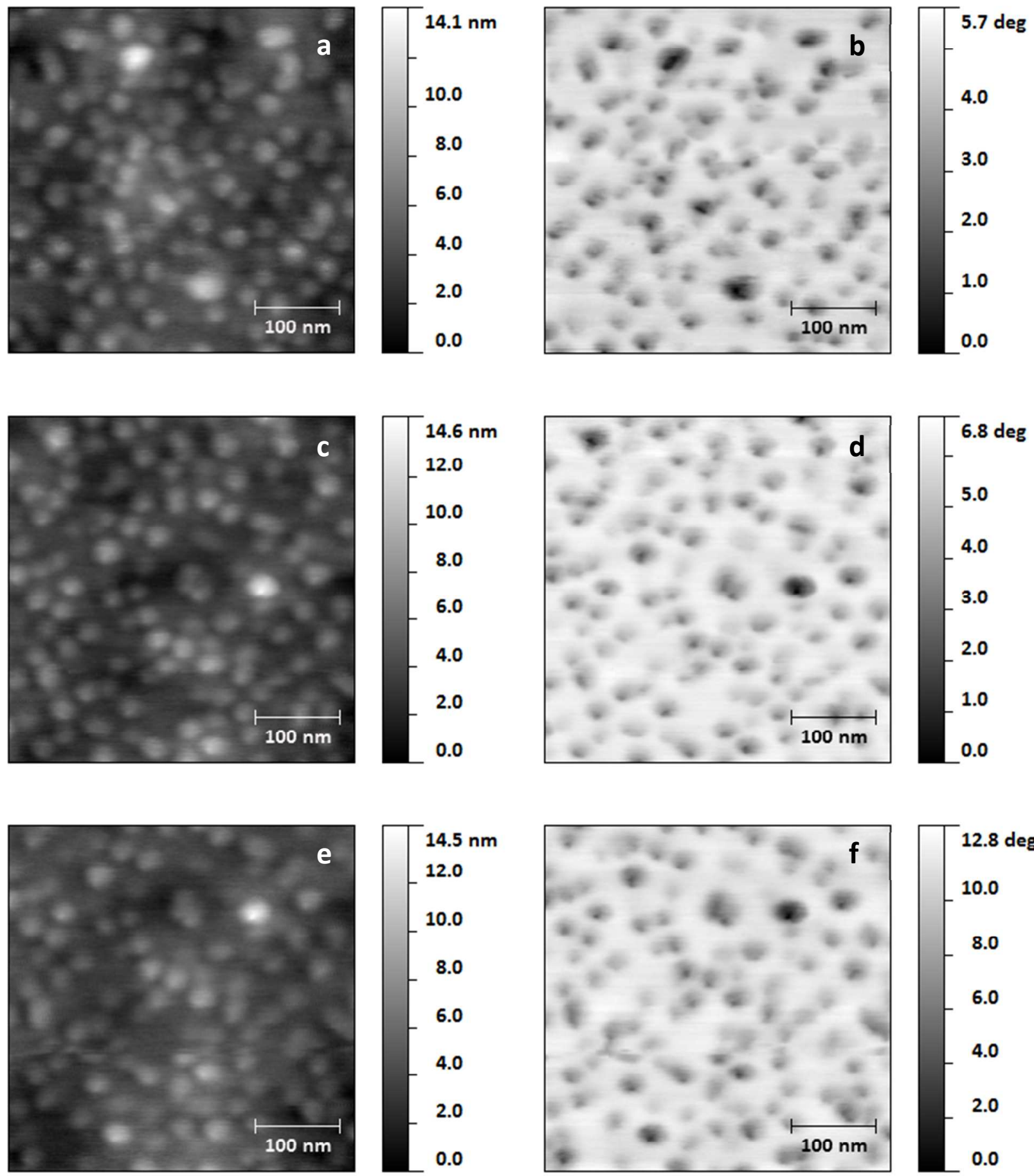

S3 AFM images of grown sample (after 12 steps of growing) analyzed by sharper tip cantilever, a, c, and e are topographic images and their corresponding phase image at $b, d$, and $f$. The cross-section views of most of the particles are less than $50 \mathrm{~nm}$ 


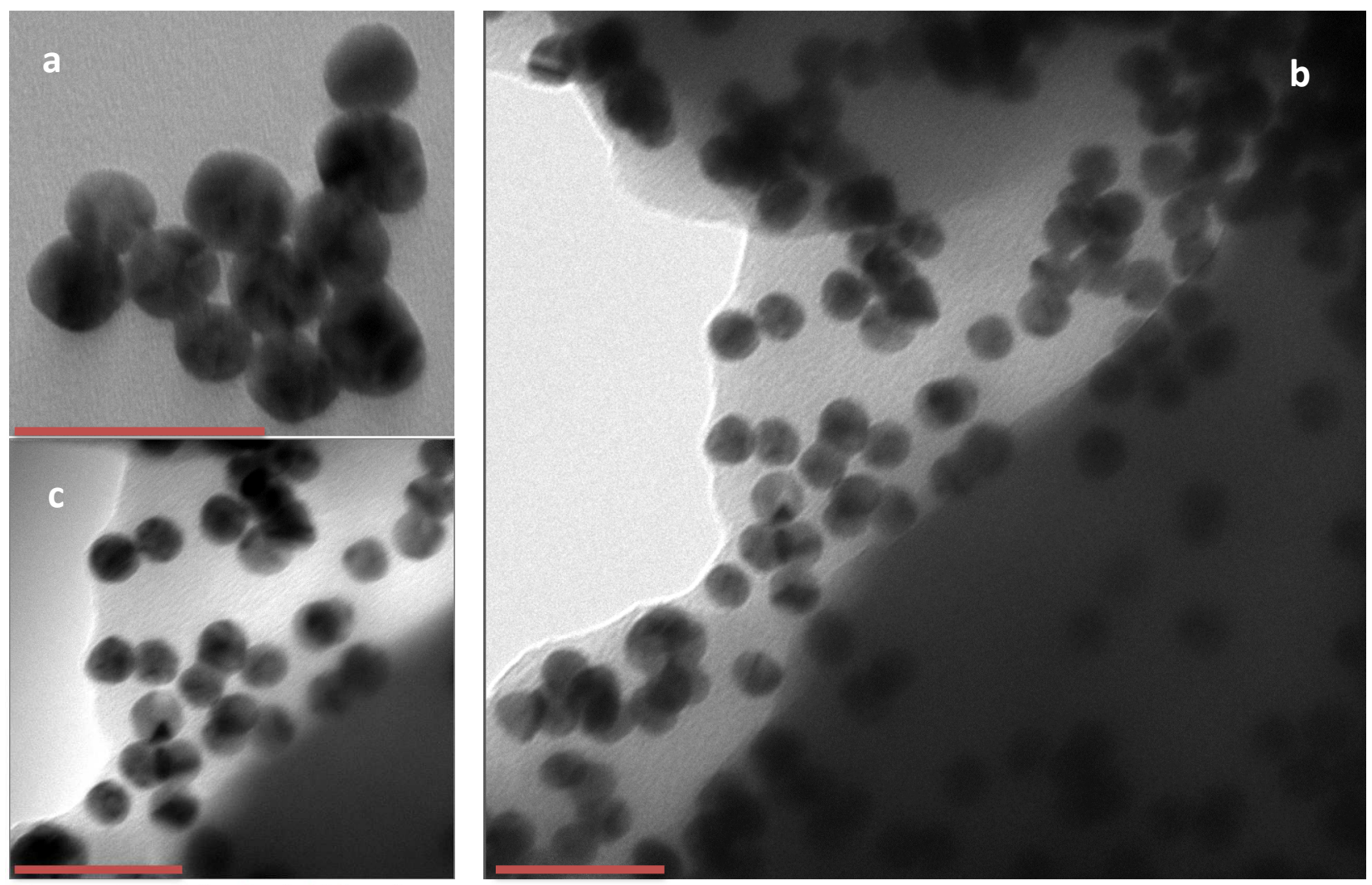

S4 TEM micrographs of colloídal seed nanoparticles at $a$, extracted from PDMS surface after deposition at $\mathrm{b}$ and $\mathrm{c}$ (scale bar 50 $\mathrm{nm})$.

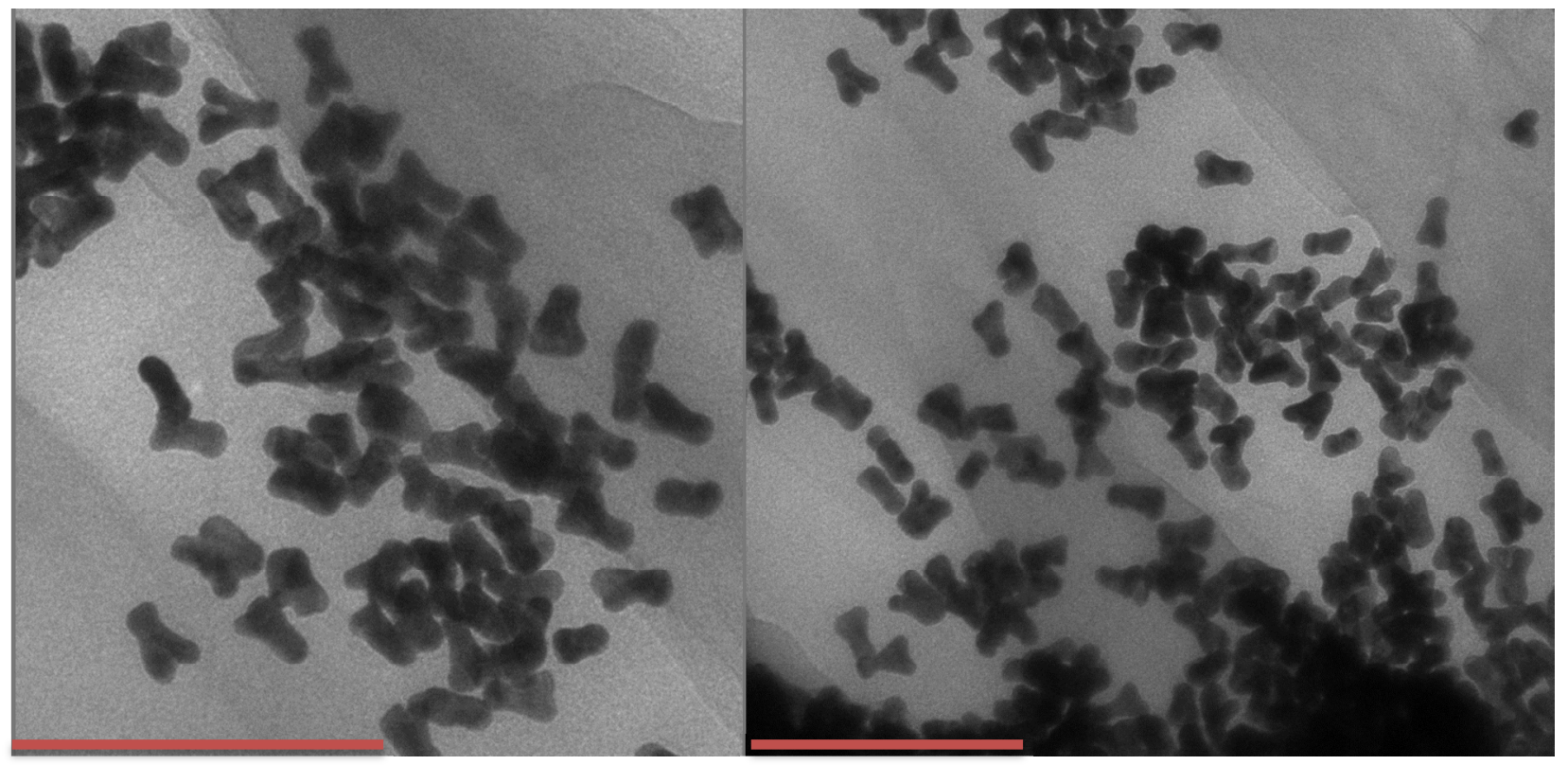

S5 TEM micrograph of extracted grown nanoparticles from PDMS surface after 7 growth steps. ${ }^{36}$ (scale bars $200 \mathrm{~nm}$ ) 